\title{
Neutronic effects of the ITER Upper Port environment update in C-Model
}

\author{
Arkady Serikov ${ }^{\mathrm{a}}$, Luciano Bertalot ${ }^{\mathrm{b}}$, Ulrich Fischera ${ }^{\mathrm{a}}$, Julio Guirao ${ }^{\mathrm{b}}$, Eduard Polunovskiy ${ }^{\mathrm{b}}$, Raul.Pampin ${ }^{\mathrm{c}}$
}

${ }^{a}$ Karlsruhe Institute of Technology (KIT), Institute for Neutron Physics and Reactor Technology, Hermann-von-Helmholtz-Platz 1, 76344 Eggenstein-Leopoldshafen, Germany ITER Organization, Route de Vinon-sur-Verdon, CS 90 046, 13067 St. Paul Lez Durance Cedex, France

Fusion for Energy (F4E)

\section{Main objectives of this work}

Development of the new 3D MCNP model of the ITER Upper Port (UP) environment integrated into the neutronics C-Model of the ITER tokamak machine.

Neutronics analysis of the newly updated C-Model Release 180430 issued on 30/04/2018 with Shut-Down Dose Rate (SDDR) calculations at 12 days of cooling. Comparison the SDDR with
previous MCNP model of ITER called C-lite V2R150304MOD. -Model in 40-degree toroidal sector symmetrically represents the whole 360 degrees of ITER machine. It is copying 9 times by using the reflective boundary conditions. as shown in the simulated 9-sector pie:
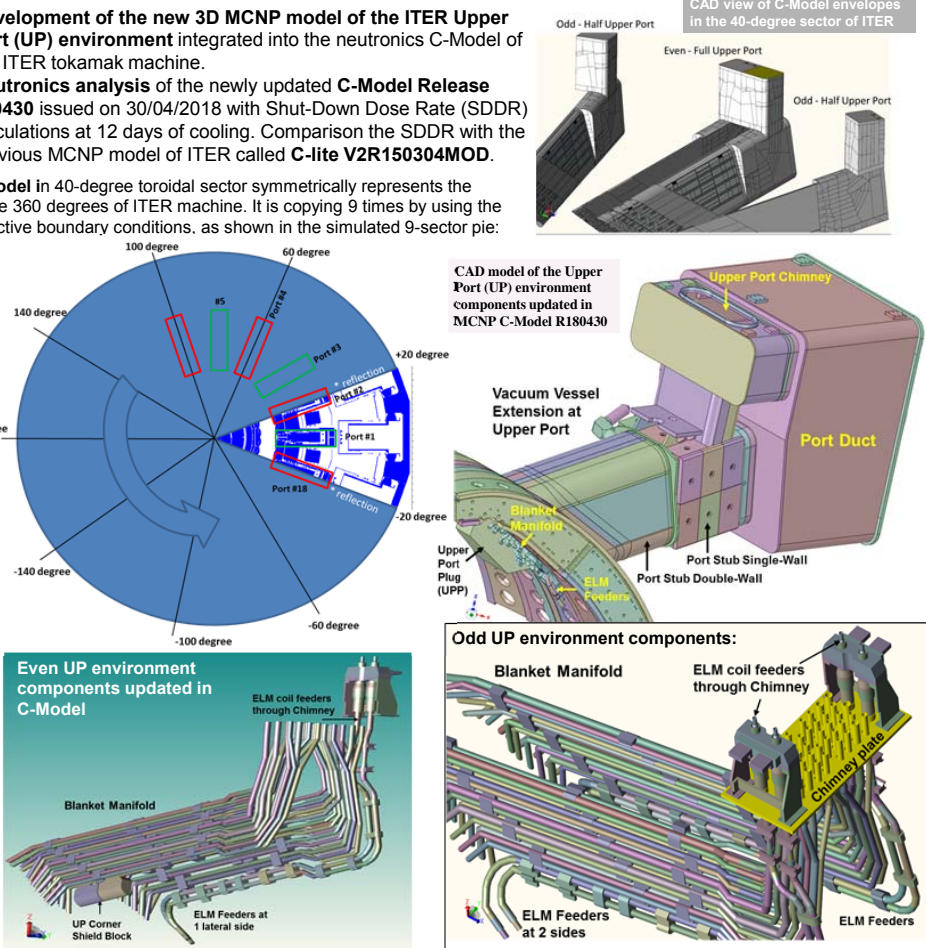

Development of the MCNP model of the UP environment

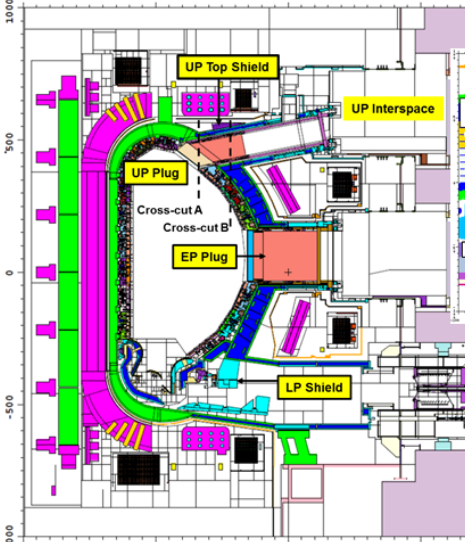

State-of-the-art codes for ITER applications: - SpaceClaim software for CAD processing;

- SuperMC (FDS, China) for CAD-to-MCNP

- Activation and SDDR calculations

FISPACT-2007 (CCFE) inventory code

R2SMesh interface (KIT) for MCNP-FISPACT

D1S-UNED v.3.1.2 for transport and SDDR

Radiation transport calculations (n/gamma fluxes)

MCNP5 v1 160, MCNP6 (LANL) code

FENDL-2.1 and 3.1 neutron cross-section libraries.

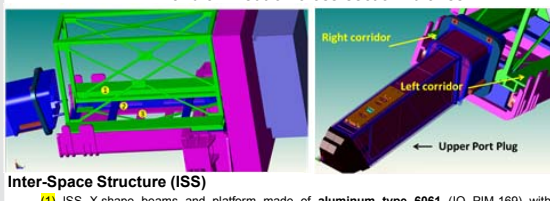
(2) ISS bogie with main structures made of steel SSM316L(N)-IG, Co 0.03 wt.\% - BLUE color (3) ISS maintenance rails made of steel SS316L(N)-10,

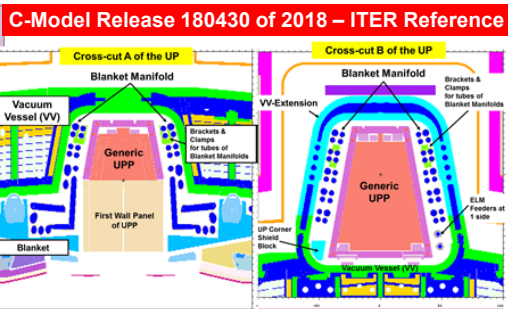

Potential shielding improvements at UP blanket manifolds, UP Stub, and UP Duct
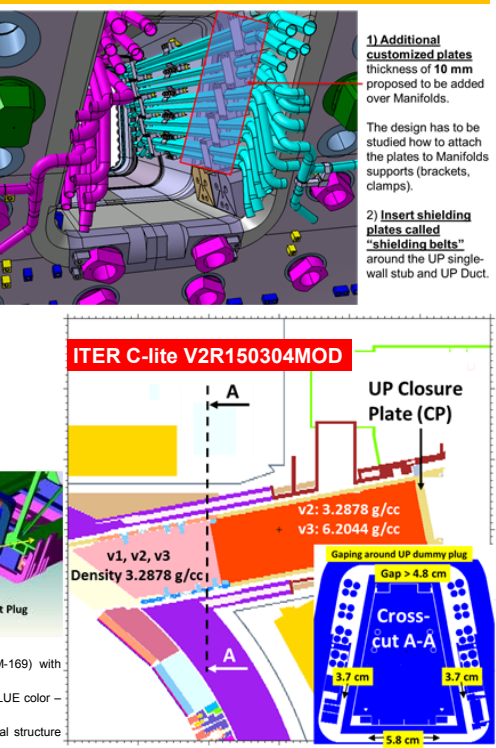

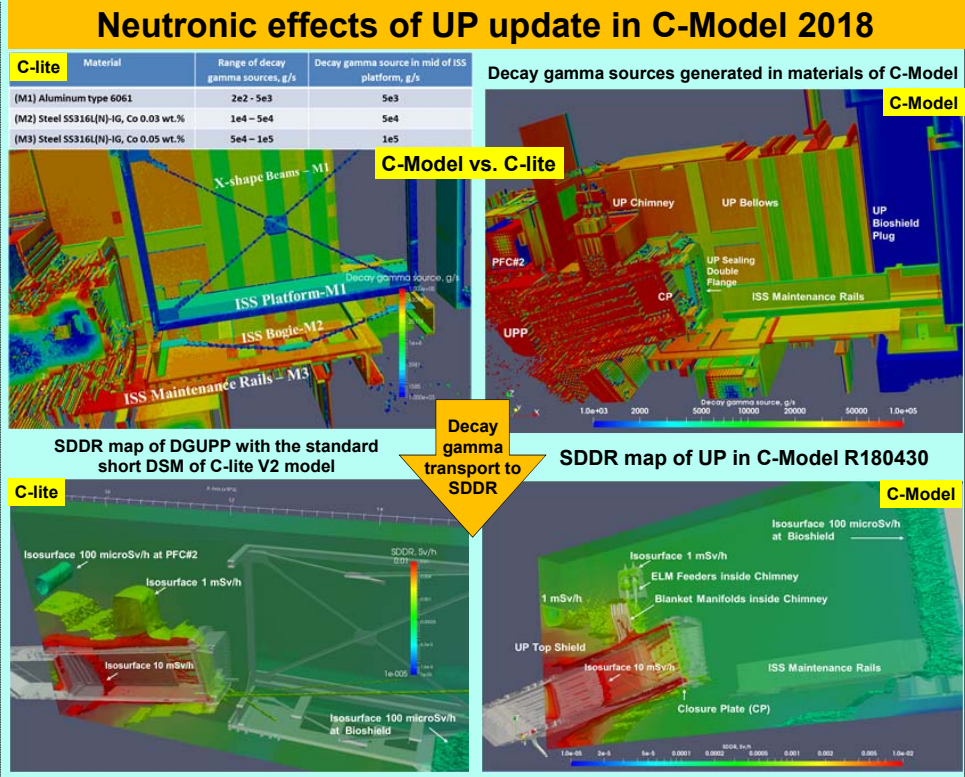

Investigation Ref. [1] the Long-DSM option and filling the UP-VV gaps in C-lite V2

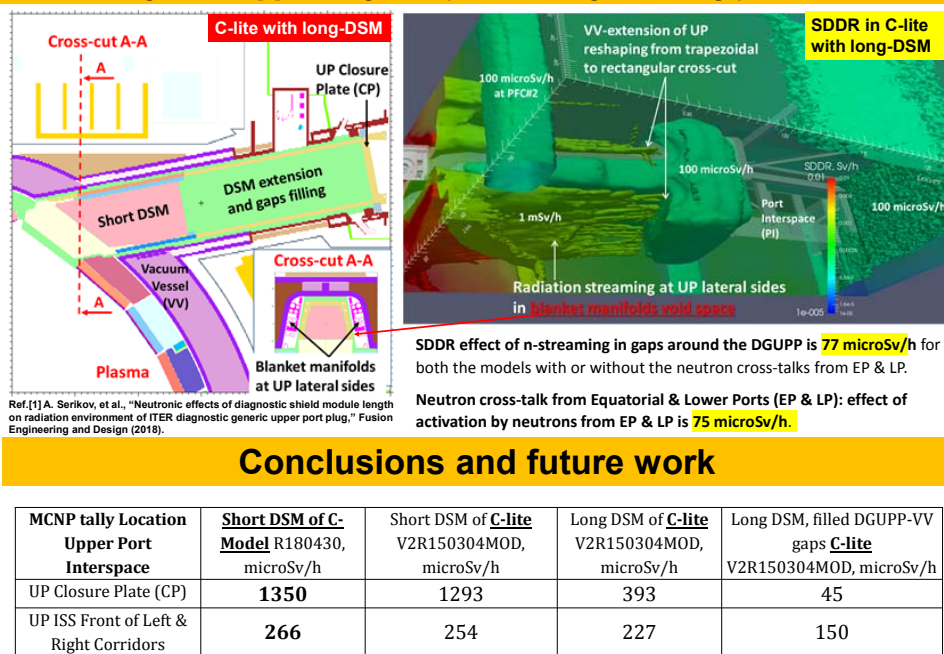

Based on the performed neutronics analysis, we conclude that the updated ITER UP environment in the MCNP C-Model Release 180430 demonstrated similar SDDR in relation to the previous version model of ITER C-lite V2R150304MOD with its short DSM Small increase by $5 \%$ of SDDR in UP interspace corridors C-Model is explained by compensating effects of additional streaming (removed shielding "cigar racks" and widening by $1.5 \mathrm{~cm}$ the gaps around UPP) and absence of radioactive sources in parts of ISS. To provide personnel access to the ISS corridors, further improvement of the shielding performance is expected by increasing the length of the DSM, in case of long-DSM the SDDR is decreased by $\sim 11 \%$ in corridors. If a design solution could be found how to fill the DGUPP-VV gaps, then reduction of $41 \%$ could be inferred, resulting $\sim 150 \mathrm{microSv} / \mathrm{h}$.

Following the ALARA principle, if it would be possible to isolate UP environment from radiation exposure from other ports and mitigate streaming inside the UPP-VV gaps, then the SDDR limit of 100 microSv/h will be met.

Engineering design development of the ITER Generic Upper Port Plug (GUPP) is still in progress. A possibility to insert shielding plates to the lateral sides of the rectangular part of UP extension is a subject of future work.

\section{Acknowledgment \& Disclaimer}

This work has been funded by the ITER Organization (IO) under the ITER service contract No. IO/17/CT/4300001478. This work was carried out using an adaption of the C-model which was developed as a collaborative effort between: AMEC Co (International), CCFE (UK), ENEA Frascati (Italy), FDS Team of INEST (PRC), ITER Organization (France), QST (Japan), KIT (Germany), UNED (Spain), University of Wisconsin-Madison (USA), F4E (Europe).

The views and opinions expressed herein do not necessarily reflect those of the ITER Organization This paper does not commit the $\mathrm{IO}$ as a nuclear operator. 